\title{
Introduction to the Special Issue on Biophotonics-Part 2
}

W ELCOME to the IEEE Journal of SElEcted TopICS IN QUANTUM ELECTRONICS (JSTQE) Special Issue on Biophotonics-Part 2! Because of significant interest in the Biophotonics field, the Editorial Board is publishing the IEEE JSTQE Special Issue on Biophotonics in two parts. Following the first JSTQE Special Issue on Biophotonics-Part 1, which was published in the May/June 2010 JSTQE Issue, we are pleased to introduce you to the JSTQE Special Issue on Biophotonics-Part 2.

The goal of this JSTQE Special Issue on Biophotonics is to provide a cross-disciplinary forum for state-of-the-art developments in biophotonics topics, including the recent progress and trends in minimally invasive biophotonics imaging, sensing, diagnostics, therapeutics, and instrumentation, as well as the emerging field of nanobiophotonics. The papers published in this issue cover a broad range of areas including:

1) advanced bioimaging;

2) multifunctional microscopy, endoscopy, and spectroscopy methods;

3) novel approaches in biophotonic diagnostics and therapeutics;

4) light-cell and light-tissue interactions;

5) novel biosensing techniques;

6) advanced nanobiophotonics;

7) novel laser, fiber-optics, and electro-optics biophotonic tools and devices.

Comprehensive overviews of the current status and future trends, as well as original results and recent developments in the field of biophotonics and nanobiophotonics will be discussed.

This issue contains 37 papers, including 18 Invited and 19 Contributed papers, authored by well-established research groups and promising scientists from all over the world. The Invited Papers include extended reviews on recent biophotonics and nanobiophotonics developments, and clinical applications in the areas of ultrahigh-resolution bioimaging; combined multifunctional microscopy, endoscopy, and spectroscopy methods; novel approaches in precancer diagnostics, photodynamic therapy, photoacoustic tomography, biosensing, and advanced methods for nanoimaging and nanobiosensing. The Contributed Papers cover a broad variety of key biophotonics research areas, including recently obtained original results on high-resolution label-free bioimaging and sensing, DNA detection, optofluidic intracavity spectroscopy, holographic microscopy, white light spectroscopy diagnostics, effective optical nerve stimulation, and development of novel biophotonics tools and devices.
The Editors hope you will find this JSTQE Special Issue on Biophotonics-Part 2 to be interesting and useful reference that will impact and stimulate the promotion of further advances in Biophotonics.

\section{ACKNOWLEDGMENT}

The Editors would like to thank the authors of all the papers in this issue for their excellent contributions and ideas, as well as many reviewers around the world, who provided high-quality reviews of the manuscripts. This issue was made possible by dedicated efforts of a number of people. The editors would like to thank the IEEE publications staff for their general support, and Ms. Chin Tan Lutz, in particular, for her prompt help, boundless energy, and excellent organization skills in helping us meet the deadline. They would also like to thank Dr. Fil Bartoli, Editor-in-Chief of the JOURNAL OF SPECIAL TOPICS IN QUANTUM ELECTRONICS, for his stimulating encouragements for this Special Issue.

\section{ILKO K. ILEV, Editor}

Center for Devices and Radiological Health

U.S. Food and Drug Administration

Silver Spring, MD 20993 USA

ilko.ilev@fda.hhs.gov

LIHONG V. WANG, Editor

Department of Biomedical Engineering

Washington University in St. Louis

St. Louis, MO 63130 USA

\section{STEPHEN A. BOPPART, Editor}

Beckman Institute for Advanced Science and Technology

Departments of Bioengineering, Electrical and Computer

Engineering, and Medicine

University of Illinois at Urbana-Champaign

Urbana, IL 61801 USA

STEFAN ANDERSSON-ENGELS, Editor

Division of Atomic Physics

Lund University

Lund, SE-22100, Sweden

BEOP-MIN KIM, Editor

Department of Biomedical Engineering

Korea University

Seoul 136-703, Korea 


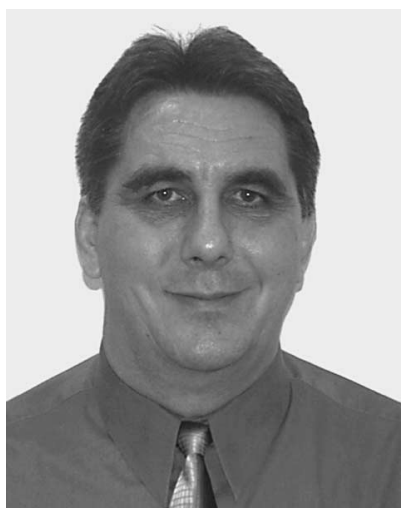

Ilko K. Ilev (M'93-SM'99) received the M.S. degree in laser physics from Sofia University, Sofia, Bulgaria, and the Ph.D. degree in laser physics from the Technical University of Sofia, Sofia, in 1983 and 1992, respectively.

He has over 20 years of experience in the United States, Europe, and Japan in the field of quantum physics and laser technologies, fiber-optics, laser medicine, noninvasive optical diagnostics and therapeutics, and biosensing and ultrahigh-resolution optical imaging. In 1995, he was with the Optoelectronics Division, Strathclyde University, Glasgow, U.K. From 1995 to 1997, he was with the Laser Technology Laboratory, Institute of Physical and Chemical Research, Tokyo, Japan. From 1998 to 2001, he was an American Academy of Science/National Research Council Research Associate in the U.S. Food and Drug Administration (FDA), Silver Spring, MD. Since May 2001, he has been with the Center for Devices and Radiological Health, FDA, where he is currently the Leader of the Optical Therapeutics and Medical Nanophotonics Laboratory. He is also an Adjunct Professor in the Department of Bioengineering, University of Maryland, College Park, MD. He is the author or coauthor of more than 300 papers published in peer-reviewed journals and has made presentations at major national and international conferences and meetings and a Guest Editor on Advanced Nanobiophotonics and Nanobiomaterials for the Nanobiotechnology Journal. His research interests include the development of novel and simple minimally invasive biophotonics and nanobiophotonics techniques, including multifunctional modalities and approaches for studying light-tissue interactions at cellular, intracellular, and molecular level, and ultrahigh-resolution bioimaging and biosensing beyond the diffraction limit in the subwavelength nanoscale range.

Dr. Ilev was an IEEE Photonics Society (formerly Lasers and Electro-Optics Society) Biophotonics Committee Chair. He has organized and chaired conferences on biophotonics and nanobiophotonics topics within the IEEE Photonics Society, the IEEE Engineering in Medicine and Biology Society, The International Society for Optical Engineers, and the Optical Society of America. $\mathrm{He}$ is an Associate Editor of the IEEE TRANSACTIONS ON BIOMEDICAL ENGINEERING, a Primary Guest Editor of the IEEE JOURNAL of SELECTED TOPICS IN QUANTUM ELECTRONICS on Biophotonics.

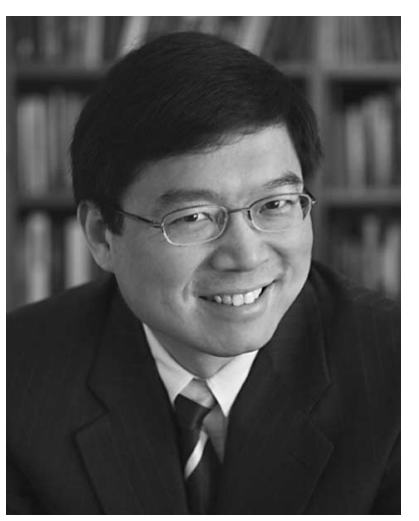

Lihong V. Wang (M'96-SM'00-F'06) received the Ph.D. degree from Rice University, Houston, TX, under the tutelage of Dr. R. Curl, Dr. R. Smalley, and Dr. F. Tittel.

$\mathrm{He}$ is currently the Gene K. Beare Distinguished Professor of biomedical engineering at Washington University, St. Louis, MO, where he is also the Director of the Optical Imaging Laboratory. He is an Editor of the first comprehensive book on photoacoustic tomography. He has authored one of the first textbooks in the field of biomedical optics. He is the Editor-in-Chief of the Journal of Biomedical Optics. He is the author or coauthor of 223 peer-reviewed journal articles and has delivered 239 invited talks. He invented or discovered frequency-swept ultrasoundmodulated optical tomography, dark-field confocal photoacoustic microscopy (PAM), optical resolution PAM, photoacoustic Doppler sensing, photoacoustic reporter gene imaging, focused scanning microwave-induced thermoacoustic tomography, exact reconstruction algorithms for photoacoustic or thermoacoustic tomography, sonoluminescence tomography, Mueller-matrix optical coherence tomography, optical coherence computed tomography, and oblique-incidence reflectometry. His Monte Carlo model of photon transport in scattering media has been used worldwide.

Prof. Wang is a Fellow of the American Institute for Medical and Biological Engineering, the Optical Society of America (OSA), and The International Society for Optical Engineers. He serves as an equal Co-Chair for the Annual Conference on Photons Plus Ultrasound, the 2010 Gordon Conference on Lasers in Medicine and Biology, and the 2010 OSA Topical Meeting on Biomedical Optics. He also Co-Chairs the International Biomedical Optics Society. He is a Chartered Member on a National Institutes of Health (NIH) Study Section. He serves as the Founding Chairs of the Scientific Advisory Boards for two companies commercializing his inventions. He was the recipient of an NIH FIRST Award, the National Science Foundation (NSF) CAREER Award, and the 2010 Joseph W. Goodman Book Writing Award. 


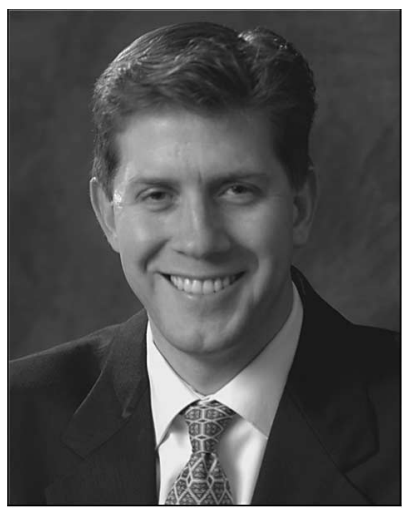

Stephen A. Boppart (S'90-M'90-SM'06) was born in Harvard, IL, in 1968. He received the B.S. degree in electrical and bioengineering and the M.S. degree in electrical engineering from the University of Illinois at Urbana-Champaign, Urbana-Champaign, in 1990 and 1991, respectively, the Ph.D. degree in electrical and medical engineering from Massachusetts Institute of Technology, Cambridge, in 1998, and the M.D. degree from Harvard Medical School, Boston, MA, in 2000.

In 2005, he completed residency training in internal medicine from the University of Illinois at Urbana-Champaign. He was a Research Scientist with the Air Force Laser Laboratory, Brooks Air Force Base, San Antonio, TX, where he was engaged in the research on developing national (American National Standards Institute) and the Air Force laser safety standards. Since 2000, he has been with the University of Illinois at Urbana-Champaign, where he is currently Professor of electrical and computer engineering, bioengineering, and medicine, and the Head of the Biophotonics Imaging Laboratory, Beckman Institute for Advanced Science and Technology. He is also the Leader of a campus-wide imaging initiative. He is the author or coauthor of more than 165 invited and contributed publications and has made over 400 invited and contributed presentations. He holds more than 25 patents filed or pending. His current research interests include the development of novel optical imaging technologies for biological and medical applications, with particular emphasis on translating these to clinical applications in cancer detection and diagnosis.

Prof. Boppart is a Fellow of the Optical Society of America and The International Society for Optical Engineers, and a member of the Society for Molecular Imaging, the Academy of Molecular Imaging, the American Association for the Advancement of Science, the American Association for Cancer Research, and the American Medical Association. He was named as one of the Top 100 Innovators in the World by the Technology Review Magazine for his research in medical technology in 2002. He was the recipient of the IEEE Engineering in Medicine and Biology Society Early Career Achievement Award in 2005.

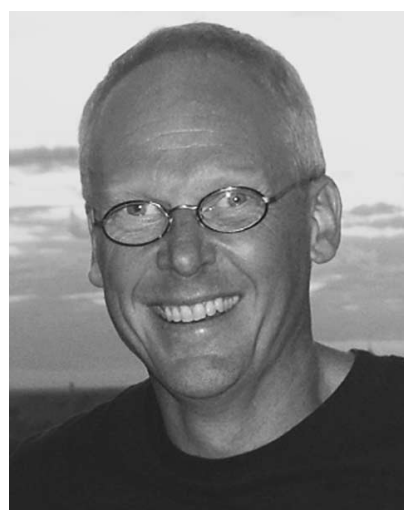

at the Island Ven, Sweden.

Stefan Andersson-Engels received the M.Sc. degree in engineering physics and the Ph.D. degree in physics from Lund University, Lund, Sweden, in 1985 and 1990, respectively.

He was involved in developing methods for tissue diagnostics based on optical spectroscopic techniques. For one year, he was with McMaster University, Hamilton, ON, Canada, where he was involved in tissue optics as well as confocal and two-photon microscopy. In 1993, he joined Lund University as an Assistant Professor, where he was an Associate Professor in 1994 and has been a Full Professor since 1999. He is the author or coauthor of more than 160 articles published in peer-reviewed journals. His current research interests include optical spectroscopy for biomedical and pharmaceutical applications, as well as interstitial photodynamic therapy of malignant tumors.

Prof. Andersson-Engels has been a Co-Organizer of several international conferences including the Gordon Conference on Lasers in Biology and Medicine in 2000, the European Conference on Biomedical Optics in 2003, and a series of biannual international summer schools in biophotonics

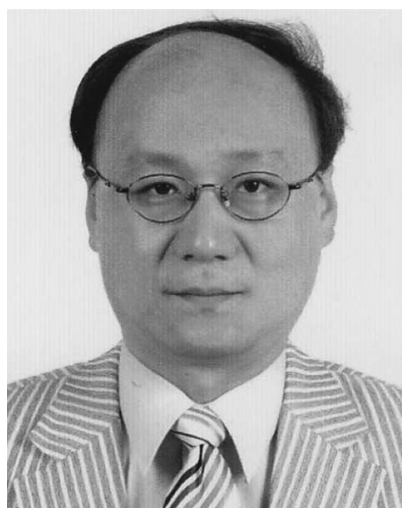

Beop-Min Kim received the B.S. degree in mechanical engineering from Korea University, Seoul, Korea, in 1989, and the M.S. and Ph.D. degrees in bioengineering from Texas A\&M University, College Station, in 1991 and 1996, respectively.

He was a Predoctoral Fellow with the University of Texas M.D. Anderson Cancer Center, Houston, and University of Texas Medical Branch, Galvaston from 1993 to 1996. From 1996 to 2001, he was with the Medical Technology Program, Lawrence Livermore National Laboratory, Livermore, CA, where he was the Staff Scientist. From 2001 to 2009, he was an Assistant/Associate Professor with the Department of Biomedical Engineering, Yonsei University, Yonsei, Korea, where was also the Department Head and the Director of the Institute of Advanced Biomedical Engineering. He is currently a Professor in the Department of Biomedical Engineering, Korea University. He is the author or coauthor of more than 40 papers in peer-reviewed journals and has made over 100 presentations at major national and international conferences and chaired many conferences on biophotonics within the Optical Society of Korea. His research interests include optical coherence tomography, near-IR spectroscopy in neuroscience, and second-harmonic generation microscopy.

Prof. Kim is currently a Board Member of the Optical Society of Korea and the Korean Society of Medical and Biological Engineering. 